\title{
A NONSTANDARD REPRESENTATION FOR BROWNIAN MOTION AND ITÔ INTEGRATION
}

\author{
BY ROBERT M. ANDERSON 1
}

Communicated by Alexandra Tulcea Bellow, October 15, 1975

A number of authors have attempted to apply Nonstandard Analysis to Probability Theory. Unfortunately, the nonstandard reformulations heretofore proposed have retained most of the essential difficulties inherent in the standard formulations. As a result, the application of nonstandard techniques has met with limited success. Hersh [4] produced a nonstandard analogue of Wiener measure. His "measure", however, is not countably additive; moreover, it is supported on a countable subset of $C([0,1])$. Using a different approach, Hersh and Greenwood [5] established some interesting results about nonstandard increments in Brownian motion and other stochastic processes, but failed to produce a successful formulation of the Itô integral or a proof of Itô's Lemma.

In a recent paper [9], Peter A. Loeb introduced a new technique for formulating probabilistic problems in nonstandard terms. He showed that any nonstandard measure space within a denumerably comprehensive enlargement could be converted into a standard measure space which inherited important structural properties from the nonstandard space. Loeb gave applications to coin tossing and the Poisson process. The present paper outlines how Brownian motion and Itô integration can be successfully treated using Loeb's technique; the details will be presented in a subsequent article.

Let $\eta$ be an infinite natural number, in the sense of nonstandard analysis. Define an internal measure space $(\Omega, \mathfrak{A}, \nu)$ by $\Omega=\{-1,1\}^{\eta}, \mathfrak{A}=\{$ internal subsets of $\Omega\}, \nu(A)=|A| / 2^{\eta}$ for $A \in \mathcal{U}$. Thus, $\nu$ is counting measure. Loeb's results show that the standard part of $\nu$ is necessarily countably additive; hence, by the Carathéodory Extension Theorem, it has a unique extension to the $\sigma$-field generated by $\mathfrak{A}$. We shall call the completion of this extension the Loeb space corresponding to $(\Omega, \mathfrak{U}, \nu)$, and denote it by $(\Omega, L(\mathfrak{U}), L(\nu))$.

Define a random walk $\chi: *[0,1] \times \Omega \rightarrow * R$ by

$$
\chi(t, \omega)=\sum_{k<[\eta t]} \frac{\omega_{k}}{\sqrt{ } \eta}+(\eta t-[\eta t]) \frac{\omega_{[\eta t]+1}}{\sqrt{ } \eta}
$$

and define $\beta:[0,1] \times \Omega \rightarrow R$ by $\beta(t, \omega)={ }^{\circ} \chi(t, \omega)$.

AMS (MOS) subject classifications (1970). Primary 60H05, 60J65, $02 \mathrm{H} 25$.

Key words and phrases. Brownian motion, stochastic integral, random walk, Stieltjes integral, Itô's Lemma, Loeb space.

${ }^{1}$ This work was supported by a Canada Council Doctoral Fellowship. 
THEOREM. (i) $\beta$ is a normalized Brownian motion; hence Brownian motion exists.

(ii) For $L(\nu)$-almost all $\omega, \chi(\cdot, \omega)$ is near-standard in $* C([0,1])$ and $\beta(\cdot, \omega)$ is continuous; hence Wiener measure exists.

The proof of this theorem is quite easy compared to the standard proofs of existence and path continuity of Brownian motion. Moreover, the intimate link between the Brownian motion $\beta$ and the random walk $\chi$ gives rise to major simplifications in the theory of Itô integration.

Let $f:[0,1] \times \Omega \rightarrow R$ be a function Itô integrable (in the standard sense) with respect to $\beta$. We can lift $f$ to an internal step function $g: *[0,1] \times \Omega \rightarrow$ $* R$. Since the random walk $\chi$ is of *-bounded variation, we can define the *. Stieltjes integral of $g$ with respect to $\chi$.

TheOREM. For $t \in[0,1], \int_{0}^{t} f(\tau, \omega) d \beta(\tau, \omega)={ }^{\circ} \int_{0}^{t} g(\tau, \omega) d \chi(\tau, \omega)$. For $L(\nu)$-almost all $\omega$, the "path" $\int_{0}^{t} g(\tau, \omega) d \chi(\tau, \omega)$, viewed as a function of $t \in *[0,1]$, is near-standard in *C([0,1]); hence, the "path" $\int_{0}^{t} f(\tau, \omega) d \beta(\tau, \omega)$, viewed as a function of $t \in[0,1]$, is continuous.

Using this theorem, we can give an all but trivial proof of Itô's Lemma. The essence of the proof is illustrated by the following argument. If we consider the nonstandard time interval $[i / \eta,(i+1) / \eta]$, with $i \in * N$, we have $d t=1 / \eta$ and $(d \chi)^{2}=( \pm 1 / \sqrt{ } \eta)^{2}=1 / \eta=d t$. Hence, the heuristic standard statement $(d \beta)^{2}=$ $d t$ is realized as an exact statement $(d \chi)^{2}=d t$ in the nonstandard theory.

The theorem also implies immediately that

$$
\int_{0}^{t} \beta(\tau, \omega) d \beta(\tau, \omega)={\left.\stackrel{[}{[\eta} \sum_{k=0}^{[}\right]-1}^{\circ} \chi(k / \eta, \omega) \omega_{k+1} \sqrt{ } \eta
$$

A simple formal manipulation of sums reduces this to ${ }^{\circ}\left(1 / 2\left(\chi^{2}(t, \omega)-t\right)\right)$; hence we obtain an easy proof that $\int_{0}^{t} \beta(\tau, \omega) d \beta(\tau, \omega)=1 / 2\left(\beta^{2}(t, \omega)-t\right)$.

\section{REFERENCES}

1. Allen R. Bernstein and Frank Wattenberg, Nonstandard measure theory, Applications of Model Theory to Algebra, Analysis, and Probability (Internat. Sympos. Pasadena, Calif., 1967), Holt, Rinehart, and Winston, New York, 1969, pp. 171-185. MR 40 \#287.

2. Patrick Billingsley, Convergence of probability measures, John Wiley, New York, 1968. MR 38 \#1718.

3. J. L. Doob, Stochastic processes, Wiley, New York; Chapman \& Hall, London, 1953. MR 15, 445 .

4. Reuben Hersh, Brownian motion and nonstandard analysis, The University of New Mexico Technical Report 277, 1973.

5. Reuben Hersh and P. Greenwood, Stochastic differentials and quasi-standard random variables (preprint).

6. Kiyosi Itô, On a formula concerning stochastic differentials, Nagoya Math. J. 3 (1951), 55-65. MR 13, 363.

7. - Stochastic integral, Proc. Imp. Acad. Tokyo 20 (1944), 519-542. MR 7, 313. 
8. S. Kakutani, Brownian motion and stochastic processes (unpublished lecture notes)

9. Peter A. Loeb, Conversion from nonstandard to standard measure spaces and applications in probability theory, Trans. Amer. Math. Soc. 211 (1975), 113-122.

10. H. P. McKean, Jr., Stochastic integrals, Probability and Math. Statist., no. 5, Academic Press, New York, 1969. MR 40 \#947.

DEPARTMENT OF MATHEMATICS, YALE UNIVERSITY, NEW HAVEN, CONNECTICUT 06520 